\title{
Modeling and Simulation of Fuze Flight Rotating Speed
}

\author{
Qiang Xiao, Zhi Yin*, Tengfei Gao, Husheng Guo, Haifei Sun, Zhipeng Wang \\ 32382 Unit of PLA, Wuhan 430311, China \\ *434629037@qq.com
}

Keywords: Fuze, Rotation Speed, Modeling.

\begin{abstract}
The ammunition fuze is the control device to control the projectile initiation in the proper position and the best time of during the flight. In view of the poor life-likeness of the current fuze rotation speed model which directly affects the centrifugal test of the fuze, so it cannot judge the quality of the fuze scientifically. In this paper the working mechanism of fuze centrifugal environment is comprehensively Analysed, the mathematical model of rotational velocity attenuation law of two kinds of projectiles constructed, and the actual measurement of projectile rotation velocity is completed based on radio technology, the motion parameters of the model calculated , and the validity of the model is verified by simulation .
\end{abstract}

\section{Introduction}

Fuze is the core device that affects the reliability and safety of ammunition, and its quality directly affects the service guarantee and combat effectiveness of ammunition[1]. The test of fuze centrifugal environment performance is an effective way to evaluate fuze quality comprehensively, and the simulation of centrifugal environment is realistic, which is directly related to the modeling of fuze flight rotation speed. Therefore, how to construct the rotating speed model of fuze effectively and scientifically to complete the centrifugal performance test of fuze has always been a key breakthrough.

Based on the characteristics of fuze flight, the working mechanism of fuze centrifugal environment is comprehensively Analysed, and the relationship between the fuze and the rotation speed of the projectile is determined. According to the rotational speed attenuation characteristics of different projectiles, the mathematical model of rotational velocity attenuation law of two kinds of projectiles constructed. Based on the radio technology, the actual measurement of the rotation speed of the projectile is completed, and the motion parameters of the mathematical model of the attenuation law are calculated, and the validity of the model is verified by simulation.

\section{Centrifugal Force Analysis of Fuze Flight}

The centrifugal safety mechanism of fuze is to realize the safety release through the rotation of fuze, and the centrifugal element is directly affected by the change of centrifugal force. During the fuze flight, the centrifugal force of the centrifugal element in the centrifugal safety mechanism is expressed as:

$$
F_{C}=2 \pi \frac{n^{2}}{60} \frac{p}{g} r
$$

\section{Mathematical model of rotating velocity of projectile}

In this paper, the mathematical modeling of rotation speed of positive ballistic flight is carried out by empirical formula. At present, the most commonly used and more accurate mathematical model of rotation speed is[2]: 


$$
w=w_{g} e^{-0.4 \frac{L D^{3}}{A} t}
$$

In the formula, for the rotational angular velocity of the projectile at time, the unit is $\mathrm{rad} / \mathrm{s}$; for the rotational angular velocity of the projectile at the time of exit; $\mathrm{D}$ for the projectile diameter, the unit $\mathrm{m}$; A for the moment of inertia of the projectile; and L for the total length of the projectile.

Usually, the trajectory of different ammunition fuzes is different in the course of external ballistic flight. According to the different law of rotation speed change and attenuation of different ammunition, the above model is modified, and its rotation speed accords with two different law of attenuation change: exponential change law and power function change law.

\subsection{The law model of power function variation}

For projectiles with large moment of inertia, such as rockets and bullets, the following positive ratios are observed:

$$
\frac{L D^{3}}{A} \propto \frac{1}{D}
$$

By substitution formula (2), we can see that the law of rotational velocity attenuation is the law of exponential change, and its expression is:

$$
w=w_{g} \exp \left(-\frac{a}{D} t\right)
$$

In the formula, a is the constant coefficient, which is related to the factors such as meteorological conditions, shooting angle, projectile structure, shape, size and elastic band, usually obtained by empirical calculation, usually:5.685 $\times 10^{-4} \sim 2.118 \times 10^{-3} \mathrm{~m} / \mathrm{s}$ 。

\subsection{Model of law of exponential change}

The model of rotation velocity change of projectile as follows:

$$
w=\frac{w_{g}}{1+b w_{g} t}
$$

In the formula, $b$ is the constant coefficient, and its variation range is $3.661 \times 10^{-6} \sim 11.653 \times 10^{-6}$, which contains the same factor as a.

\section{Centrifugal Force Analysis of Fuze Flight}

in order to realize the above mathematical model motion parameters a and b calculation, it is still necessary to obtain the actual measurement data of the projectile. At present, there are many methods for measuring the rotation speed of projectile. The common methods are mechanical measurement, imaging measurement and azimuth sensor[3-5]. Here, using radio technology to complete the measurement of the actual rotation speed of the projectile, only need to install radio fuze on the projectile, which has the advantages of relatively simple measurement process, low cost and high safety. The system block diagram for measuring the rotation speed of the projectile using radio technology is shown in fig. 1. 


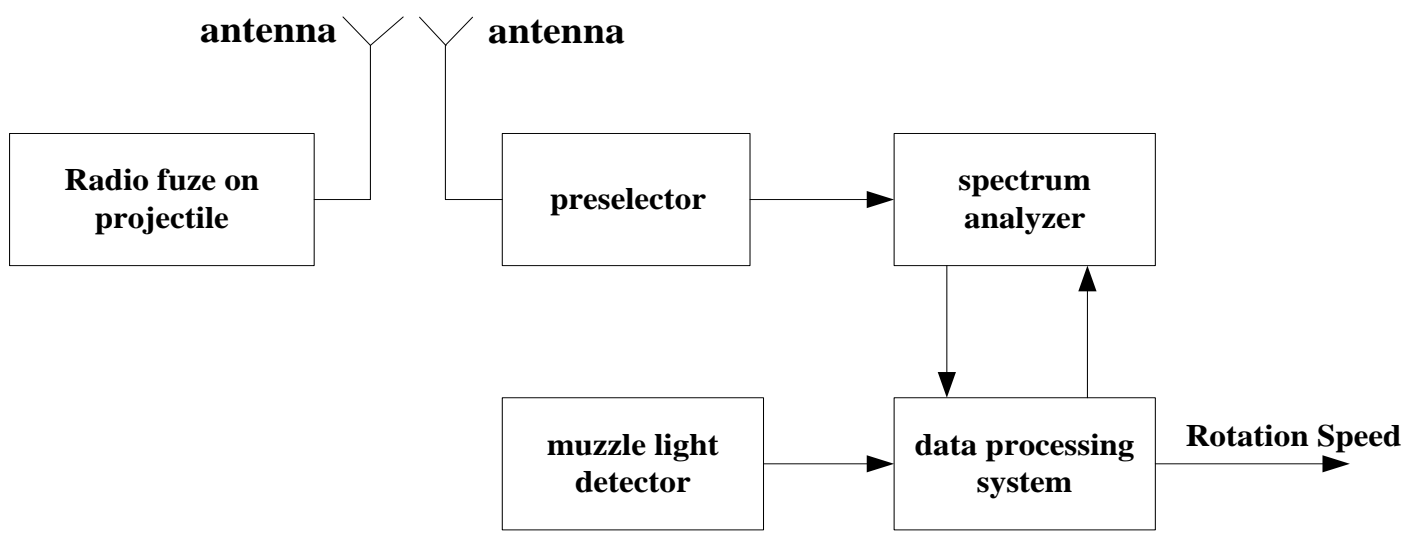

Fig. 1Measuring rotation speed of projectile by radio technology

\section{Simulation Validation}

\subsection{Calculation of Motion Parameters}

According to the measured velocity of projectile rotation, the values of constant $a$ and $b$ in the formula can be calculated. The expression is:

$$
\left\{\begin{array}{l}
\mathrm{a}=\frac{D}{\mathrm{t}} \ln \left(\frac{w_{g}}{w}\right) \\
\mathrm{b}=\frac{1}{w_{g}}\left(\frac{w_{g}}{w}-1\right) \frac{1}{t}
\end{array}\right.
$$

Due to the inevitable errors in the measurement process, the more accurate values of $a$ and $b$ are obtained by obtaining the average values of the same projectile for multiple groups of measured data.

According to the actual measured value of the rotation speed of the projectile and the maximum value of the rotation speed of the projectile at the time of the projectile production, the minimum value of the design, the table of rotational motion parameters of the two typical fuzes is given, as shown in Table 1.

Table 1. Rotating Motion Parameters for Typical Fuzes

\begin{tabular}{ccccc}
\hline Fuze Type & AttenuationType & Max-speed & Design -Mini & a or b constant \\
\hline Some rocket fuze & power function & 1000 & 985 & $6.389 \times 10^{-4}$ \\
Some grenade fuze & index number & 2920 & 1000 & $10.932 \times 10^{-6}$ \\
\hline
\end{tabular}

\subsection{Model validation}

a) Power function simulation

For a rocket fuze, the rotational angular velocity of the projectile $w_{g}$ is $1000 \mathrm{rad} / \mathrm{s}$, a is $4.685 \times$ $10^{-4}$, and $\mathrm{D}$ is $0.13 \mathrm{~m}$, and the simulation results are shown in Fig.2. 


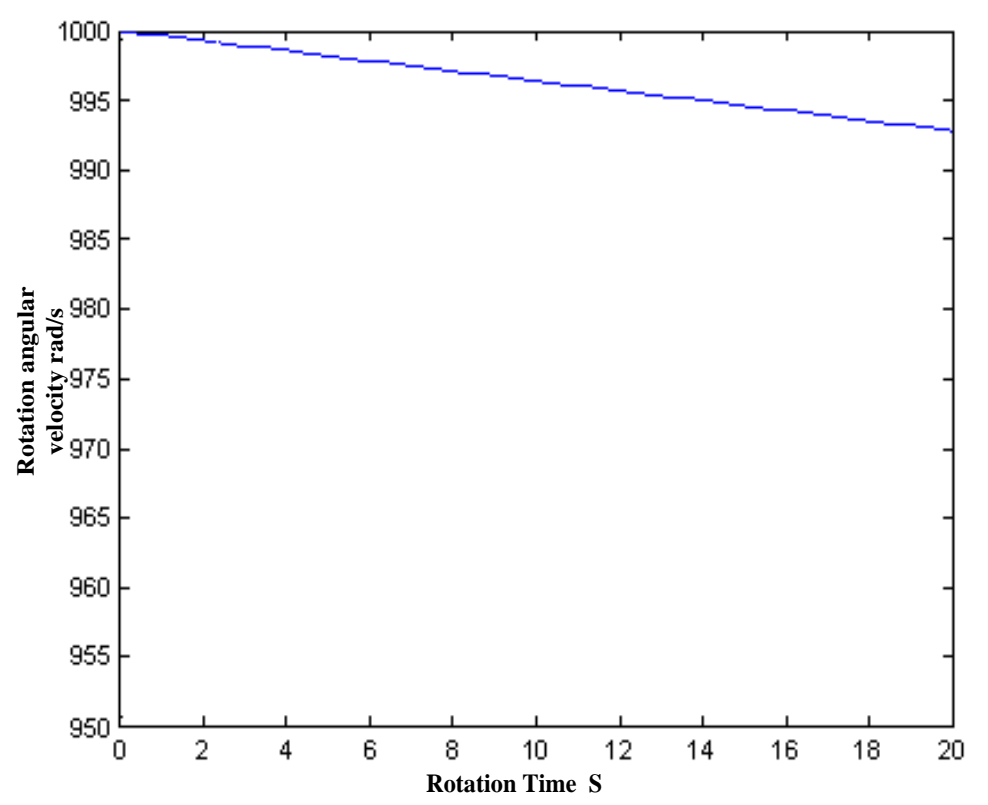

Fig. 2 Variation of rotational angular velocity of a rocket fuze

b) law of exponential change simulation

For a grenade fuze, the rotational angular velocity of the projectile out of the chamber $w_{g}$ is $2920 \mathrm{rad} / \mathrm{s}$, and b takes $10.932 \times 10^{-6}$. the rotational angular velocity variation of the projectile is shown in figure 3.

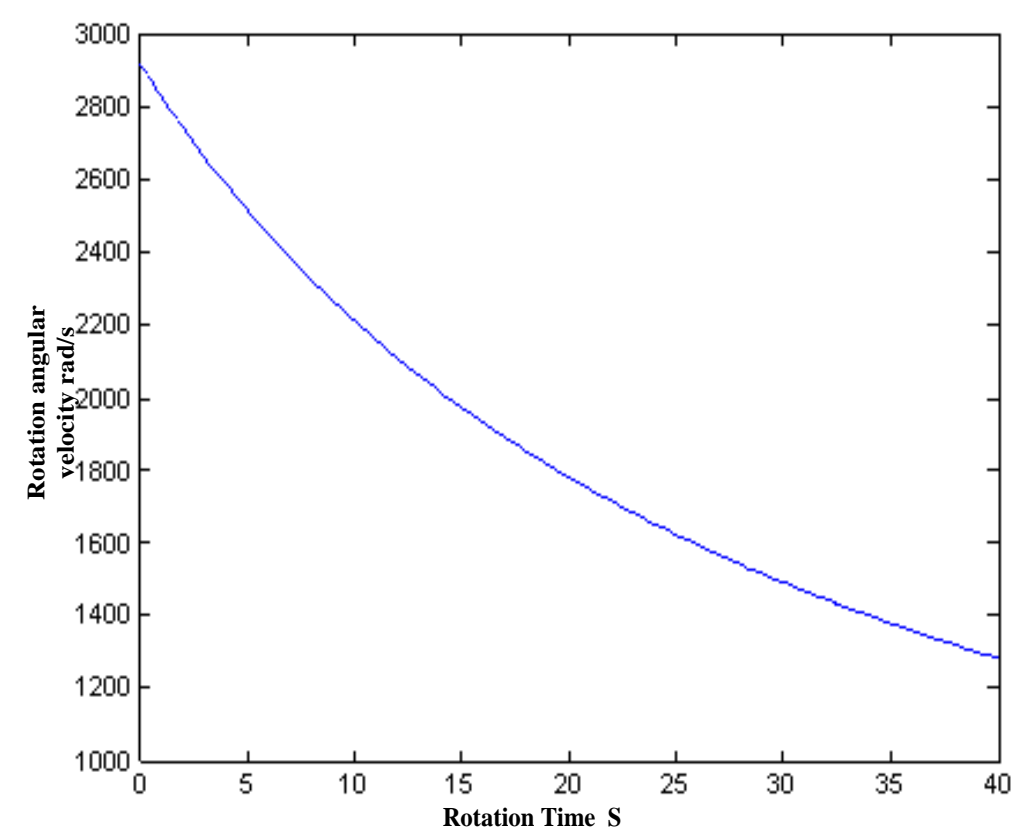

Fig. 3 Variation of rotational angular velocity of a grenade fuze

\section{Conclusion}

In this paper, the rotation speed model of fuze and projectile is constructed, and the validity and scientificity of the model are verified by simulation. The research results can provide reference for the design of fuze centrifugal environment simulation and detection system. in the next step of the study, the best method will be sought to obtain more close to the practical model to ensure the realistic rotation speed of the fuze. 


\section{References}

[1]He Zhang,Haojie Li. Fuze Mechanism[M]. Beijing:Beijing Institute of Technology Press,2013,p.3-18.

[2]Yushi Wang. Semi-empirical formulae for the law of velocity attenuation of rotational projectile from the outer projectile [J]. Journal of Detection and Control,Vol. 42(2003)No.1,p.1-6.

[3]Jumin Huang,Lijun Yang,et al. A study on continuous wave Doppler measurement of rotating velocity of shells[J].Journal of the Chinese Academy of Electronic Sciences,Vol. 4(2009)No.3,p.304-307.

[4]Weijuan Xin,KeningWang.A triangular method for measuring projectile speed [J]. Development and Application,(2009)No.1,p.31-33.

[5]Jiayu Sun. Application of Radio Fuze for Measuring the Speed of Long Path projectile [J] .Journal of Beijing Institute of Technology,Vol. 21(2001)No.2,p.243-247. 